\title{
Treatment and Outcomes in Differentiated Thyroid Cancer: A Retrospective Exploration in Three UK Centres That Provide Different Advice on Low lodine Diets
}

Clare England ( $\nabla$ clare.england@bristol.ac.uk)

University of Bristol

Kate Ingarfield-Herbert

University of Bristol

Matthew Beasley

University Hospitals Bristol NHS Foundation Trust

\section{Laura Moss}

Velindre Cancer Centre

Sobhan Vinjamuri

Royal Liverpool and Broadgreen University Hospital NHS Trust

Ingrid Haupt-Schott

Velindre Cancer Centre

\section{Gail McKane}

Royal Liverpool and Broadgreen University Hospital NHS Trust

\section{Linda Hunt}

University of Bristol

\section{Georgia Herbert}

University of Bristol

\section{Sam Leary}

University of Bristol

\section{Andy Ness}

University of Bristol

\section{Charlotte Atkinson}

University of Bristol

\section{Research Article}

Keywords: Thyroid cancer, Radioiodine ablation, Retrospective studies, Low lodine Diet

Posted Date: January 27th, 2021

DOl: https://doi.org/10.21203/rs.3.rs-140980/v1 
License: (우 This work is licensed under a Creative Commons Attribution 4.0 International License. Read Full License 


\section{Abstract}

Background International guidelines on the treatment of differentiated thyroid cancers promote low iodine diets (LID) before radioiodine remnant ablation. Evidence that the LID ultimately improves treatment success is inconsistent. This study aimed to determine if there is a difference in ablation success rates according to provision of advice to follow a LID.

Methods Retrospective study of patients with differentiated thyroid cancer treated with total thyroidectomy and radioiodine remnant ablation between 01/01/2015 and 31/12/2016 in 3 centres advising: no LID (C1: $n=108)$; LID for 1-week before (C2: $n=50)$; LID for 2-weeks before and 48 hours (C3: n=59) after RRA. Response to treatment was determined by adapted American Thyroid Association Dynamic Risk Stratification Score, stratified as excellent, indeterminate, or incomplete response.

Results In total, 217 patients were included in the analysis. We found differences in preparation for radioiodine remnant ablation and in the assessment of outcomes between centres. Furthermore, although there was little difference in staging between centres there was a difference in the percentage of patients receiving 1.1GBq vs higher administered activities (15\% in C1, 22\% in C2 and 44\% in C3, p<0.001). An excellent response was recorded for $49 \%$ in $\mathrm{C} 1,48 \%$ in $\mathrm{C} 2$ and $36 \%$ in $\mathrm{C} 3(\mathrm{p}=0.61)$. With $\mathrm{C} 1$ as reference group, the odds ratios (OR) for an excellent response were C2 OR: 0.96 (95\% Cl 0.46,2.00) and C3 OR: 0.62 (95\% Cl 0.29,1.30), p=0.40.

Conclusions We found no evidence that advice to follow a low iodine diet for 1 or 2 weeks before radioiodine remnant ablation impacts on ablation success but differences between centres means the results should be regarded as exploratory. There is no immediate need to change practice regarding the LID, but a prospective multi-centre study with a more homogenous approach to patient management or a randomised controlled trial will provide more definitive recommendations.

\section{Background}

Globally, over 255,000 new cases of thyroid cancer were recorded in 2017 , with $70 \%$ in women(1). A third of cases live in higher-income countries, but the age standardised incidence rate is increasing fastest in middleincome countries. In 2017 in the UK there were 1068 new cases of thyroid cancer in men and 2756 in women compared with 310 in men and 815 in women in 1993(2). Increasing incidence of thyroid cancer is a global trend, although mortality is, in most regions, falling(1).

Differentiated thyroid cancer (DTC) accounts for $95 \%$ of cases and has 10 -year survival rates of $80-90 \%(3)$. DTC is usually treated with total thyroidectomy, which may be followed by treatment with radioactive iodine $\left({ }^{131} \mathrm{I}\right)$ to destroy residual thyroid or cancerous tissue. 'Radioiodine remnant ablation' (RRA) denotes destruction of residual thyroid tissue whereas 'radioiodine therapy' refers to ${ }^{131} \mathrm{I}$ administration for known residual local or metastatic disease(3). The decision on whether to proceed with ${ }^{131} \mathrm{I}$, and the administered activity, is determined, in part, by staging and histological features. Depending on these factors, patients are classified into three groups 1) definite indications for RRA (large tumour size or extensive extra thyroidal extension or distant metastases) 2) uncertain indications 3 ) no indication (small tumour size, no extra thyroidal extension, minimally invasive, welldifferentiated). The decision to proceed or not is individualised for patients in group 2, taking into account 
factors such as tumour size, histology, lymph node involvement and full extent of any extra thyroidal extension and after discussion of risks and benefits of RRA with the patient(3).

Thyroid stimulating hormone (TSH) is raised to facilitate uptake of ${ }^{131} \mathrm{I}$. This used to be achieved through thyroid hormone withdrawal (THW) but, since approval in the mid-2000s, recombinant human TSH (rhTSH) is routinely used(4). Non-radioactive iodine competes with ${ }^{131}$ I for uptake, which may reduce efficacy of RRA(5). In relation to iodine status at the time of RRA, the European Association of Nuclear Medicine Therapy Committee recommends aiming for a urine iodine concentration (UIC) of $<100 \mathrm{mcg} / \mathrm{l}$ and postponing RRA if UIC $>150$ $200 \mathrm{mcg} / \mathrm{I}(5)$. International guidelines state that iodinated contrast agents should be avoided in the 8 weeks before RRA, to avoid RRA if amiodarone has been taken within the previous 12 months and, based on expert opinion, recommend a low iodine diet (LID) for 1 to 2 weeks prior to RRA(3-6). LID advice reduces iodine intake and iodine status in patients with high iodine intake $(7,8)$. However, no randomised controlled trials have been conducted on whether LIDs improve ablation success, and evidence from cohort and retrospective studies is mixed $(9,10)$, particularly in countries with low dietary iodine, such as the UK(11).

Despite acknowledging the lack of evidence for LIDs, British Thyroid Association guidelines advise an LID prior to RRA(3). In the UK, many treatment centres use the UK Low lodine Diet Working Group diet sheet and advise restricting fish, seafood, seaweed, dairy produce, eggs, iodised salt (although salt is not routinely iodised in the UK) and a wide variety of foods containing these ingredients as well as nutritional supplements and cough mixture(12). However, different treatment centres give differing advice, particularly regarding length of dietary restriction and when to restart usual diet(13). The effect of LID advice on treatment success in the UK has not been examined.

This is a retrospective study of patients with DTC comparing response to RRA in three UK centres that give different LID advice prior to RRA. The aim is to determine if there is a difference in response according to provision of advice to follow a LID.

\section{Methods}

\section{Sites}

We recruited three UK treatment centres that routinely administer RRA for thyroid cancer giving different LID advice. Centre 1 (C1) did not advise patients to follow a LID, Centre 2 (C2) advised a LID for one week prior to RRA and Centre 3 (C3) advised a LID for two weeks prior to RRA and 48 hours post.

\section{Patients}

Clinical records of patients diagnosed with DTC who underwent RRA for thyroid cancer at the three sites between 01/01/2015 and 31/12/2016 were reviewed. Eligible patients were those treated with one stage or two stage total thyroidectomy and diagnosed with papillary or follicular thyroid cancer by histology. Included patients were those with stages $\mathrm{N} 0$ to $\mathrm{N} 1 \mathrm{~b}$ and treated with any administered activity who had been prepared with either rhTSH or THW protocol. Records were excluded if patients had distant metastases, were experiencing recurrence, or had two radioiodine therapies planned from the outset due to residual disease or local involvement of surrounding tissue. Patients diagnosed during 2016 but treated with RRA during 2017 were excluded. Identification of thyroid cancer during treatment for other cancers was not an exclusion criterion. 


\section{Outcome}

Response to treatment was determined by a three tier system advised by UK Guidelines for the Management of Thyroid Cancer(3), adapted from the American Thyroid Association Dynamic Risk Stratification Score (ATA Score)(14) which uses results from neck ultrasound (US) and thyroglobulin (Tg) tests at 9 to 12 months post ablation. Responses are classified as excellent response (low risk of recurrence), indeterminate response (intermediate risk of recurrence) and incomplete response (high risk of recurrence). Where neck US was not available, results from diagnostic whole-body scan (DxWBS) was used(3). Thyroglobulin is secreted by normal and cancerous thyroid cells and detectable Tg post-thyroidectomy indicates remnant thyroid tissue or the presence of residual or recurrent tumour(3). Tg may be measured using immunometric assays ( $\mathrm{Tg}-\mathrm{IA}$ ) or radioimmunoassays ( $\mathrm{Tg}-\mathrm{RIA})$. $\mathrm{Tg}$ antibodies $(\mathrm{TgAb})$ can interfere with the measurement of $\mathrm{Tg}$, producing falsely low results and masking disease. Both Tg-IA and Tg-RIA are subject to false negatives, but no TgAb detection cut-off exists that eliminates false negatives and false positives(15). Patients with no evidence of disease on neck US or DxWBS, supressed or stimulated $\mathrm{Tg}<1 \mathrm{ug} / \mathrm{l}$ and any detectable $\mathrm{TgAb}$ were classified as an indeterminate response, in strict accordance with the ATA Score.

\section{Data extraction}

Anonymised data on demographics, details of surgery, tumour, node and metastasis (TNM) staging, ${ }^{131}$ I dose, post ablation assessment and evaluation of treatment success at 9 to 12 months post RRA were extracted. Data extraction was undertaken and checked by clinical staff or trained coders.

\section{Statistical analysis}

Overall comparisons were made between centres with respect to patients' ages, gender, grouped TNM stage (7th edition)(16), ${ }^{131} \mathrm{I}$ administered activity, and ATA Score, using chi-squared tests or one-way ANOVA as appropriate. These were followed by within-centre comparisons of these variables between patients with excellent, indeterminate, or incomplete outcomes.

Across all centres, numbers of people experiencing an incomplete response were small so indeterminate/incomplete responses were combined. The main analysis used logistic regression to compare centres in respect of excellent response, adjusting successively for age and sex and then administered activity of ${ }^{131} \mathrm{I}$ and TNM stage; coding for response was incomplete/indeterminate $=0$ vs excellent $=1$, therefore higher odds were associated with better outcomes. A sensitivity analysis was undertaken with a graded response, using ordered logistic regression (proportional odds), coding the response as incomplete $=1$, indeterminate $=2$, excellent $=3$. For both the main analysis and sensitivity analysis C1 was the comparison group .

\section{Results}

A total of 289 records were screened, 217 were eligible $(C 1=108, C 2=50, C 3=59)$. Distant metastasis $(n=30$ [42\%]) and recurrence $(n=18[25 \%])$ were the most common reasons for ineligibility. Differences were observed between centres regarding preparation for RRA and tests used to assess treatment success. C2 and C3 prepared patients using rhTSH, assessed success with neck US and measured Tg using Beckman Tg-IA for all cases(17). In contrast only 9 patients in $\mathrm{C} 1$ were assessed through neck US and Tg-IA measures (Roche E170). However, C3 was less likely to use supressed $\mathrm{Tg}$ measures than $\mathrm{C} 1$ and $\mathrm{C} 2$. These differences were unexpected and the initial objective to determine if there was a difference in treatment success according to provision of LID advice was 
hampered by this. The results provided below are therefore considered exploratory in nature, and any differences observed between centres may not be due to differences in LID advice.

\section{Descriptive comparison between centres}

Table 1 shows between-centre comparisons in respect of age, gender, TNM stage, ${ }^{131}$ I administered activity and the results of post ablation whole body scans (PaWBS). C1 had fewer patients with TNM stages III/IV than the other centres, but there was little statistical evidence of a difference between centres $(p=0.14)$. A difference was observed between ${ }^{131} \mathrm{I}$ administered activity above 1.1GBq; fewer patients in $\mathrm{C} 3$ received higher activities $(\mathrm{P}<$ 0.001). PaWBS indicated that four (4\%) patients in $\mathrm{C} 1$ and four $(7 \%)$ in $\mathrm{C} 3$ showed uptake in cervical nodes. Within-centre differences in response failed to show overall differences between subgroups (except possibly for C3 (TNM stage and administered activity), but numbers were small) (Supplementary tables). 
Table 1

- Between centre comparisons of patient and tumour characteristics and response to treatment

\begin{tabular}{|c|c|c|c|c|}
\hline Centre & $\begin{array}{l}\text { Centre } 1 \\
\text { (Col. \%) }\end{array}$ & $\begin{array}{l}\text { Centre } 2 \\
\text { (Col. \%) }\end{array}$ & $\begin{array}{l}\text { Centre } 3 \\
\text { (Col. \%) }\end{array}$ & $\begin{array}{l}\text { p-value (Chi-squared } \\
\text { unless indicated) }\end{array}$ \\
\hline Total patients & 108 & 50 & 59 & - \\
\hline \multicolumn{5}{|l|}{ Age at diagnosis (years) } \\
\hline Mean (SD) & $\begin{array}{l}50.0 \\
(15.3)\end{array}$ & $\begin{array}{l}44.5 \\
(15.0)\end{array}$ & $\begin{array}{l}49.7 \\
(17.7)\end{array}$ & $0.12^{1}$ \\
\hline$\leq 45$ & $42(41 \%)$ & $27(54 \%)$ & $26(44 \%)$ & 0.32 \\
\hline$>45$ & $60(59 \%)$ & $23(46 \%)$ & $33(56 \%)$ & \\
\hline \multicolumn{5}{|l|}{ Sex } \\
\hline Male & $37(34 \%)$ & $17(34 \%)$ & $18(31 \%)$ & 0.88 \\
\hline Female & $71(66 \%)$ & $33(66 \%)$ & $41(70 \%)$ & \\
\hline \multicolumn{5}{|l|}{ TNM stage } \\
\hline I/II & $66(73 \%)$ & $30(60 \%)$ & $34(60 \%)$ & 0.14 \\
\hline III/IV & $24(27 \%)$ & $20(40 \%)$ & $23(40 \%)$ & \\
\hline \multicolumn{5}{|l|}{ Administered activity ${ }^{131}$ I (GBq) } \\
\hline 1.1 & $16(15 \%)$ & $11(22 \%)$ & $26(44 \%)$ & $<0.001^{2}$ \\
\hline 3.7 & $89(82 \%)$ & 39 (78\%) & $33(56 \%)$ & \\
\hline 5.5 & $2(2 \%)$ & $0(0 \%)$ & $0(0 \%)$ & \\
\hline 7.4 & $1(1 \%)$ & $0(0 \%)$ & $0(0 \%)$ & \\
\hline \multicolumn{5}{|l|}{ Post ablation whole body scan } \\
\hline $\begin{array}{l}\text { Uptake in neck (unspecified or in keeping } \\
\text { with residual thyroid tissue) }\end{array}$ & $97(90 \%)$ & $41(82 \%)$ & $52(88 \%)$ & 0.001 \\
\hline No uptake & 0 & 0 & $2(3 \%)$ & \\
\hline Uptake in cervical nodes & $4(4 \%)$ & 0 & $4(7 \%)$ & \\
\hline
\end{tabular}

Centre $1=$ no low iodine diet (LID) advice; Centre $2=1$ week LID advice; Centre $3=2$ week LID advice and 48 hours after ablation

ATA = American Thyroid Association; Col \% = column percentage; $\mathrm{LID}=$ low iodine diet; $\mathrm{SD}=$ standard deviation; $\mathrm{TgAb}=$ thyroglobulin antibodies; TNM = cancer staging (tumour, node, metastasis)

${ }^{1}$ means compared with one-way ANOVA

2administered activity $1.1 \mathrm{vs} \geq 3.7 \mathrm{Gbq}$ in Centre 1 to facilitate chi-squared analysis

${ }^{3}$ complete responders vs combined Indeterminate/Incomplete 


\begin{tabular}{|c|c|c|c|c|}
\hline Centre & $\begin{array}{l}\text { Centre } 1 \\
\text { (Col. \%) }\end{array}$ & $\begin{array}{l}\text { Centre } 2 \\
\text { (Col. \%) }\end{array}$ & $\begin{array}{l}\text { Centre } 3 \\
\text { (Col. \%) }\end{array}$ & $\begin{array}{l}\text { p-value (Chi-squared } \\
\text { unless indicated) }\end{array}$ \\
\hline Other / unclear & $3(3 \%)$ & $7(14 \%)$ & 0 & \\
\hline \multicolumn{5}{|l|}{ ATA score } \\
\hline Excellent response & $52(49 \%)$ & $24(48 \%)$ & $21(36 \%)$ & 0.61 \\
\hline Indeterminate & $45(43 \%)$ & $22(44 \%)$ & $31(53 \%)$ & $\left(0.26^{3}\right)$ \\
\hline Incomplete & $9(9 \%)$ & $4(8 \%)$ & $6(10 \%)$ & \\
\hline \multicolumn{5}{|c|}{$\begin{array}{l}\text { Centre } 1=\text { no low iodine diet (LID) advice; Centre } 2=1 \text { week LID advice; Centre } 3=2 \text { week LID advice and } 48 \\
\text { hours after ablation }\end{array}$} \\
\hline \multicolumn{5}{|c|}{$\begin{array}{l}\text { ATA = American Thyroid Association; Col \% = column percentage; } \mathrm{LID}=\text { low iodine diet; } \mathrm{SD}=\text { standard } \\
\text { deviation; } \mathrm{TgAb}=\text { thyroglobulin antibodies; TNM = cancer staging (tumour, node, metastasis) }\end{array}$} \\
\hline \multicolumn{5}{|c|}{${ }^{1}$ means compared with one-way ANOVA } \\
\hline \multicolumn{5}{|c|}{ 2administered activity $1.1 \mathrm{vs} \geq 3.7 \mathrm{Gbq}$ in Centre 1 to facilitate chi-squared analysis } \\
\hline \multicolumn{5}{|c|}{${ }^{3}$ complete responders vs combined Indeterminate/Incomplete } \\
\hline
\end{tabular}

\section{Differences in response}

Centre differences in response are shown in Table 2. Although the response at C3 appeared worse than at other centres, the confidence intervals around the odds ratio (OR) were wide and there was no good evidence for a difference between centres, with or without adjustment for known confounders, i.e., age, gender, and TNM stage and activity of ${ }^{131}$ I (C2 OR: 0.96 (95\% Cl 0.46,2.00); C3 OR: 0.62 (95\% Cl 0.29,1.30), p = 0.40). The sensitivity analysis, with graded response, showed similar results (C2 OR: 0.96 (95\% Cl 0.47,1.95); C3 OR $0.64(95 \% \mathrm{Cl}$ $0.32,1.30)$. 
Table 2

- Logistic regression to compare centres in respect of excellent response (vs combined indeterminate/incomplete)* assessed using the ATA Score

\begin{tabular}{|c|c|c|c|c|c|}
\hline $\begin{array}{l}\text { Centre (overall \% with } \\
\text { excellent response, } n=214 \text { ) }\end{array}$ & $\begin{array}{l}\text { Unadjusted } \\
\mathbf{N}=214\end{array}$ & $\begin{array}{l}\text { Minimally } \\
\text { adjusted } \\
\text { (age** and } \\
\text { sex) } \\
\mathrm{N}=208\end{array}$ & $\begin{array}{l}\text { Partially } \\
\text { adjusted } \\
\text { (age, sex, } \\
\text { dose*** of } \\
{ }^{131} \text { I) } \\
\mathrm{N}=208\end{array}$ & $\begin{array}{l}\text { Partially } \\
\text { adjusted } \\
\text { (age, sex, } \\
\text { TNM } \\
\text { stage) } \\
\mathrm{N}=194\end{array}$ & $\begin{array}{l}\text { Fully adjusted } \\
\text { (age, sex, } \\
\text { TNM stage } \\
\text { and dose of } \\
131 \text { D) } \\
N=194\end{array}$ \\
\hline & $\begin{array}{l}\text { OR }(95 \% \\
\mathrm{Cl})\end{array}$ & $\begin{array}{l}\text { OR }(95 \% \\
\mathrm{Cl})\end{array}$ & OR $(95 \% \mathrm{Cl})$ & $\begin{array}{l}\text { OR }(95 \% \\
\mathrm{Cl})\end{array}$ & OR $(95 \% \mathrm{Cl})$ \\
\hline Centre 1 (49\%) & 1 (Ref.) & 1 (Ref.) & 1 (Ref.) & 1 (Ref.) & 1 (Ref.) \\
\hline Centre 2 (48\%) & $\begin{array}{l}0.96 \\
(0.49,1.88)\end{array}$ & $\begin{array}{l}1.01 \\
(0.50,2.02)\end{array}$ & $\begin{array}{l}1.03 \\
(0.51,2.08)\end{array}$ & $\begin{array}{l}0.92 \\
(0.45,1.91)\end{array}$ & $\begin{array}{l}0.96 \\
(0.46,2.00)\end{array}$ \\
\hline Centre 3 (36\%) & $\begin{array}{l}0.59 \\
(0.31,1.14)\end{array}$ & $\begin{array}{l}0.60 \\
(0.31,1.16)\end{array}$ & $\begin{array}{l}0.66 \\
(0.33,1.33)\end{array}$ & $\begin{array}{l}0.56 \\
(0.28,1.14)\end{array}$ & $\begin{array}{l}0.62 \\
(0.29,1.30)\end{array}$ \\
\hline Overall p-value & 0.26 & 0.26 & 0.45 & 0.25 & 0.40 \\
\hline \multicolumn{6}{|c|}{$\begin{array}{l}\text { Centre } 1=\text { no low iodine diet (LID) advice; Centre } 2=1 \text { week LID advice; Centre } 3=2 \text { week LID advice and } 48 \\
\text { hours after ablation }\end{array}$} \\
\hline \multicolumn{6}{|l|}{ Ref = reference group } \\
\hline \multicolumn{6}{|c|}{$\begin{array}{l}\text { *coding for response in this analysis incomplete/indeterminate }=0 \text {, excellent }=1 \text {, therefore higher odds } \\
\text { indicate more favourable outcome }\end{array}$} \\
\hline \multicolumn{6}{|l|}{ **Continuous } \\
\hline \multicolumn{6}{|c|}{$\star \star \star 5.5 \mathrm{Gbq}$ and $7.4 \mathrm{Gbq}$ combined } \\
\hline$\star \star \star \star$ Likelihood-ratio test & & & & & \\
\hline
\end{tabular}

\section{Discussion}

This retrospective study of patients with DTC compared treatment response rates at 9 to 12 months post RRA from three centres in the UK that gave different LID advice prior to RRA. We found differences between centres in both preparation and determination of treatment success, which was not expected at the outset of this study. Comparison between centres was thus hampered, and findings should be treated as exploratory.

We found no evidence for a difference in ablation success at 9 to 12 months assessment between the centres, adjusting for age, sex, TNM stage and administered activity ${ }^{131} \mathrm{I}$. Treatment response for C3 (2-week LID) appeared worse, with a $40 \%$ reduction in the odds of experiencing an excellent response compared to that of experiencing an excellent response at C1 (no LID). However, the confidence interval was wide and included no difference and when an outcome is common the odds ratio will tend to overestimate underlying risk. There was no difference in effect size between C1 (no LID advice) and C2 (1-week LID). 
Only $45 \%$ of included patients were classified as experiencing an excellent response at 9 to 12 months post RRA, which appears low. Ablation success was determined using the ATA Score combining results of neck US or DxWBS with stimulated or supressed Tg measurements(3). Patients with TgAb were not removed from the analysis but, in accordance with the guidelines, were classified as having an 'indeterminate' response. However, results from DxWBS or neck US alone indicate that no abnormality was detected for $84 \%$ of patients (data not shown), which is comparable with success rates reported in other studies, including the Hi Lo trial(18).

\section{Comparison with other LID studies}

No randomised controlled trials have examined whether LID advice prior to RRA or radioiodine therapy improves treatment success rates in thyroid cancer. In 1983 Maxon et al(19) observed that use of a LID increased ${ }^{131}$ I administered activity to the tumour during diagnostic scans (LID = 19 vs normal diet $=21$ ), but evidence that the LID improved treatment success was lacking. Since then, retrospective examinations of patient records have been conducted to determine whether use of LID improves success rates or whether iodine status is associated with treatment success. Table 3 summarises relevant studies. Only Pluijmen et al(20) found that there was a difference in success rates between patients advised to follow a LID and those who were not. However, between

studies there is variation in preparation, ${ }^{131} \mathrm{I}$ administered activity, how response to treatment is assessed and whether patients with detectable TgAb were excluded or not. 
Table 3

- Summary of studies examining the impact of low iodine dietary advice or iodine status on radioiodine therapy success rates

\begin{tabular}{|c|c|c|c|c|c|c|}
\hline $\begin{array}{l}\text { Author, year, } \\
\text { country }\end{array}$ & Study design & $\begin{array}{l}\text { Preparation; } \\
\text { administered } \\
\text { activity }\end{array}$ & Comparisons & $\begin{array}{l}\text { lodine } \\
\text { status at } \\
\text { radioiodine } \\
\text { therapy }\end{array}$ & $\begin{array}{l}\text { Treatment } \\
\text { success } \\
\text { (\% } \\
\text { successful) }\end{array}$ & Comments \\
\hline $\begin{array}{l}\text { Morris et al, } \\
2001 \\
\text { USA }\end{array}$ & $\begin{array}{l}\text { Retrospective } \\
\text { comparison } \\
\text { of cohorts } \\
\text { treated } \\
\text { between } \\
1990-1994 \\
\text { vs 1997- } \\
1999 \\
\text { Total } n=92\end{array}$ & $\begin{array}{l}\text { THW } \\
3.7 \mathrm{GBq} \text { or } \\
5.5 \mathrm{GBq}\end{array}$ & $\begin{array}{l}\text { LID advice } \\
\text { for } 10-14 \\
\text { days prior to } \\
\text { RRA. } \\
\text { Less } \\
\text { stringent LID } \\
\text { (avoid fish, } \\
\text { seafood and } \\
\text { iodine } \\
\text { supplements) } \\
\text { ( } n=50) \\
\text { Stringent LID } \\
\text { ( } n=42 \text { ) }\end{array}$ & $\begin{array}{l}\text { Less } \\
\text { stringent } \\
\text { LID }(\mathrm{n}=7 \\
\text { healthy } \\
\text { volunteers) } \\
\text { vs } \\
\text { Stringent } \\
\text { LID }(\mathrm{n}=7) \\
\text { Mean (sd) } \\
\text { UIC } \\
381(196) \\
\text { mcg/L vs } \\
174(128) \\
\text { mcg/L }\end{array}$ & $\begin{array}{l}\text { Assessed } \\
\text { by DxWBS } \\
\text { alone } \\
\text { Less } \\
\text { stringent } \\
\text { LID vs } \\
\text { Stringent } \\
\text { LID } \\
62 \% \text { vs } \\
68 \%, p= \\
0.53\end{array}$ & $\begin{array}{l}\text { Higher } \\
\text { success } \\
\text { rates were } \\
\text { observed at } \\
\text { higher } \\
\text { administered } \\
\text { activity in } \\
\text { both groups. } \\
\text { Although } \\
\text { more } \\
\text { patients in } \\
\text { the stringent } \\
\text { LID group } \\
\text { were treated } \\
\text { with } 3.7 \mathrm{GBq} \\
\text { than } 5.5 \mathrm{GBq} \text {, } \\
\text { subgroup } \\
\text { sample sizes } \\
\text { were too } \\
\text { small to } \\
\text { provide } \\
\text { evidence } \\
\text { that the LID } \\
\text { improved } \\
\text { success } \\
\text { rates at } \\
\text { lower doses. }\end{array}$ \\
\hline $\begin{array}{l}\text { Pluijmen et } \\
\text { al, } 2003 \\
\text { Netherlands }\end{array}$ & $\begin{array}{l}\text { Retrospective } \\
\text { comparison } \\
\text { of cohorts } \\
\text { treated } \\
\text { between } \\
1986-1991 \\
\text { vs 1992- } \\
1998 \\
\text { Total n = } 120 \\
\text { Excluding } \\
\text { patients with } \\
\text { TgAbs }\end{array}$ & $\begin{array}{l}\text { THW } \\
2.9 \mathrm{GBq}\end{array}$ & $\begin{array}{l}\text { No LID } \\
\text { advice ( } \mathrm{n}= \\
61 \text { ) } \\
\text { LID for } 4 \\
\text { days prior to } \\
\text { RRA, only } \\
\text { patients with } \\
24-U I E< \\
50 \text { mcg/day } \\
\text { included ( } \mathrm{n}= \\
59 \text { ) }\end{array}$ & $\begin{array}{l}\text { No LID } \\
\text { advice (n = } \\
9 \text { ) vs LID } \\
\text { advice (n = } \\
59) \\
\text { Mean (sd) } \\
24-U I E \\
159(9.0) \\
\text { mcg/day } \\
\text { vs } 27 \\
(11.6) \\
\text { mcg/day }\end{array}$ & $\begin{array}{l}\text { Assessed } \\
\text { by DxWBS } \\
\text { plus Tg } \\
\text { No LID vs } \\
\text { LID } \\
\\
48 \% \text { vs } \\
65 \% \text { p } \\
0.001\end{array}$ & $\begin{array}{l}\text { A LID that } \\
\text { reduces } \\
\text { iodine status } \\
\text { to }< \\
50 \text { mcg/day } \\
\text { improves } \\
\text { efficacy } \\
\text { radioiodine } \\
\text { therapy. }\end{array}$ \\
\hline $\begin{array}{l}\text { Tala Jury et } \\
\text { al, } 2010 \\
\text { Italy }\end{array}$ & $\begin{array}{l}\text { Retrospective } \\
\text { cohort } \\
\text { treated } \\
\text { between } \\
\text { 1998-2008 }\end{array}$ & $\begin{array}{l}\operatorname{rhTSH}(\mathrm{n}= \\
76) \\
\mathrm{THW} \\
125)\end{array}$ & $\begin{array}{l}\text { No specific } \\
\text { LID advice, } \\
\text { patients } \\
\text { advised to } \\
\text { avoid iodine }\end{array}$ & $\begin{array}{l}\text { Mean UIC } \\
(n=201) \\
132(160) \\
m c g / L\end{array}$ & $\begin{array}{l}\text { Assessed } \\
\text { by DxWBS } \\
\text { alone ( } \mathrm{n}= \\
201)\end{array}$ & $\begin{array}{l}\text { No } \\
\text { difference in } \\
\text { mean UIC } \\
\text { between } \\
\text { those who }\end{array}$ \\
\hline $\begin{array}{l}\text { DxWBS = Dia } \\
\text { remnant abla } \\
\text { Thyroglobulir } \\
\text { concentration }\end{array}$ & $\begin{array}{l}\text { nostic whole bo } \\
\text { on; rhTSH = Rec } \\
\text { antibodies; THV } \\
\text { Ul/Cr = Urine ioc }\end{array}$ & mbinant hum & 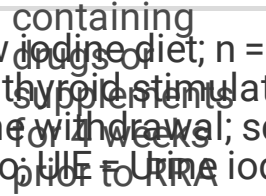 & $\begin{array}{l}\text { umber; OR = } \\
\text { ig hormone; } \\
\text { = Standard de } \\
\text { ne excretion }\end{array}$ & $\begin{array}{l}\text { ds ratio; RR } \\
=\text { Thyroglob } \\
\text { ation; UIC = }\end{array}$ & 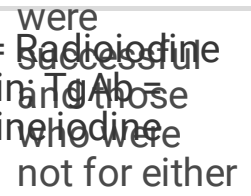 \\
\hline
\end{tabular}




\begin{tabular}{|c|c|c|c|c|c|c|}
\hline $\begin{array}{l}\text { Author, year, } \\
\text { country }\end{array}$ & Study design & $\begin{array}{l}\text { Preparation; } \\
\text { administered } \\
\text { activity }\end{array}$ & Comparisons & $\begin{array}{l}\text { lodine } \\
\text { status at } \\
\text { radioiodine } \\
\text { therapy }\end{array}$ & $\begin{array}{l}\text { Treatment } \\
\text { success } \\
\text { (\% } \\
\text { successful) }\end{array}$ & $\begin{array}{l}\text { definnition of } \\
\text { Sơcesesnts } \\
\text { No } \\
\text { difference } \\
\text { between }\end{array}$ \\
\hline & Total $\mathrm{n}=201$ & $\begin{array}{l}\text { 1.1 GBq to } \\
5.5 \mathrm{GBq}\end{array}$ & & & $\begin{array}{l}85 \% \\
\text { success for } \\
\text { total group }\end{array}$ & $\begin{array}{l}\text { rhTSH and } \\
\text { THW for } \\
\text { success } \\
\text { rates or } \\
\text { mean UIC. }\end{array}$ \\
\hline
\end{tabular}

Stratified

by UIC

(mcg/L)

$<50$ ( $\mathrm{n}=$

41) $88 \%$

50-100 (n

= 54) $82 \%$

101-150

$(n=47)$

$81 \%$

151-200

$(n=25)$

$85 \%$

200-250

$(\mathrm{n}=17)$

$88 \%$

$>250(\mathrm{n}=$

17) $82 \%$

Assessed

by DxWBS

plus $\mathrm{Tg}$,

excluding

DxWBS = Diagnostic whole body scan; $\mathrm{LID}=\mathrm{Low}$ iodine diet; $\mathrm{n}=$ number; $\mathrm{OR}=$ Odds ratio; RRA = Radioiodine remnant ablation; rhTSH = Recombinant human thyroid stimulating hormone; $\mathrm{Tg}=$ Thyroglobulin; $\mathrm{TgAb}=$ Thyroglobulin antibodies; THW = thyroid hormone withdrawal; sd = Standard deviation; UIC = Urine iodine concentration; $\mathrm{UI} / \mathrm{Cr}=$ Urine iodine creatinine ratio; $\mathrm{UIE}=$ Urine iodine excretion 


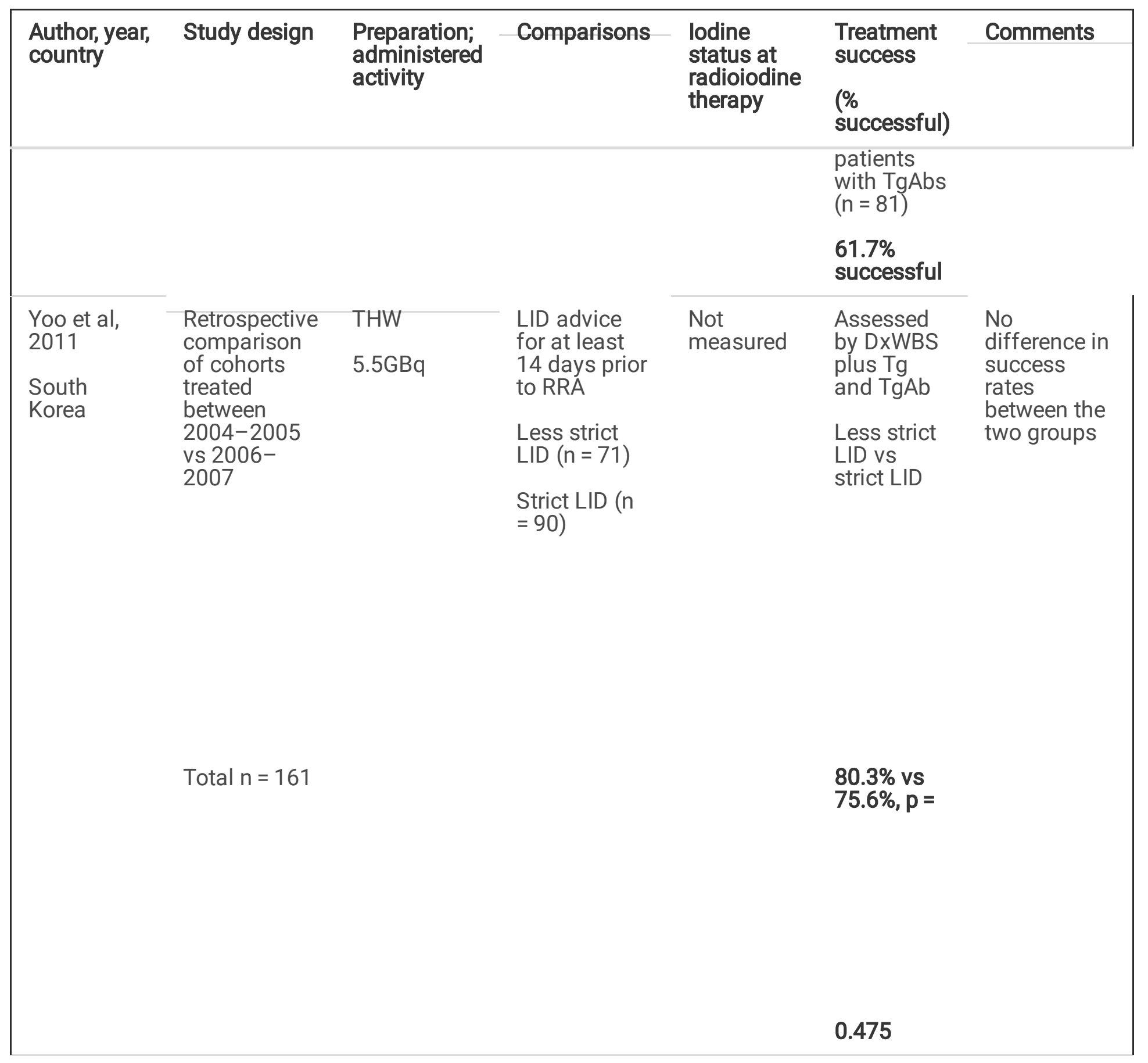

DxWBS = Diagnostic whole body scan; $\mathrm{LID}=\mathrm{Low}$ iodine diet; $\mathrm{n}=$ number; $\mathrm{OR}=$ Odds ratio; $\mathrm{RRA}=$ Radioiodine remnant ablation; rhTSH = Recombinant human thyroid stimulating hormone; $\mathrm{Tg}=$ Thyroglobulin; $\mathrm{TgAb}=$ Thyroglobulin antibodies; THW = thyroid hormone withdrawal; sd = Standard deviation; UIC = Urine iodine concentration; $\mathrm{UI} / \mathrm{Cr}=$ Urine iodine creatinine ratio; $\mathrm{UIE}=$ Urine iodine excretion 


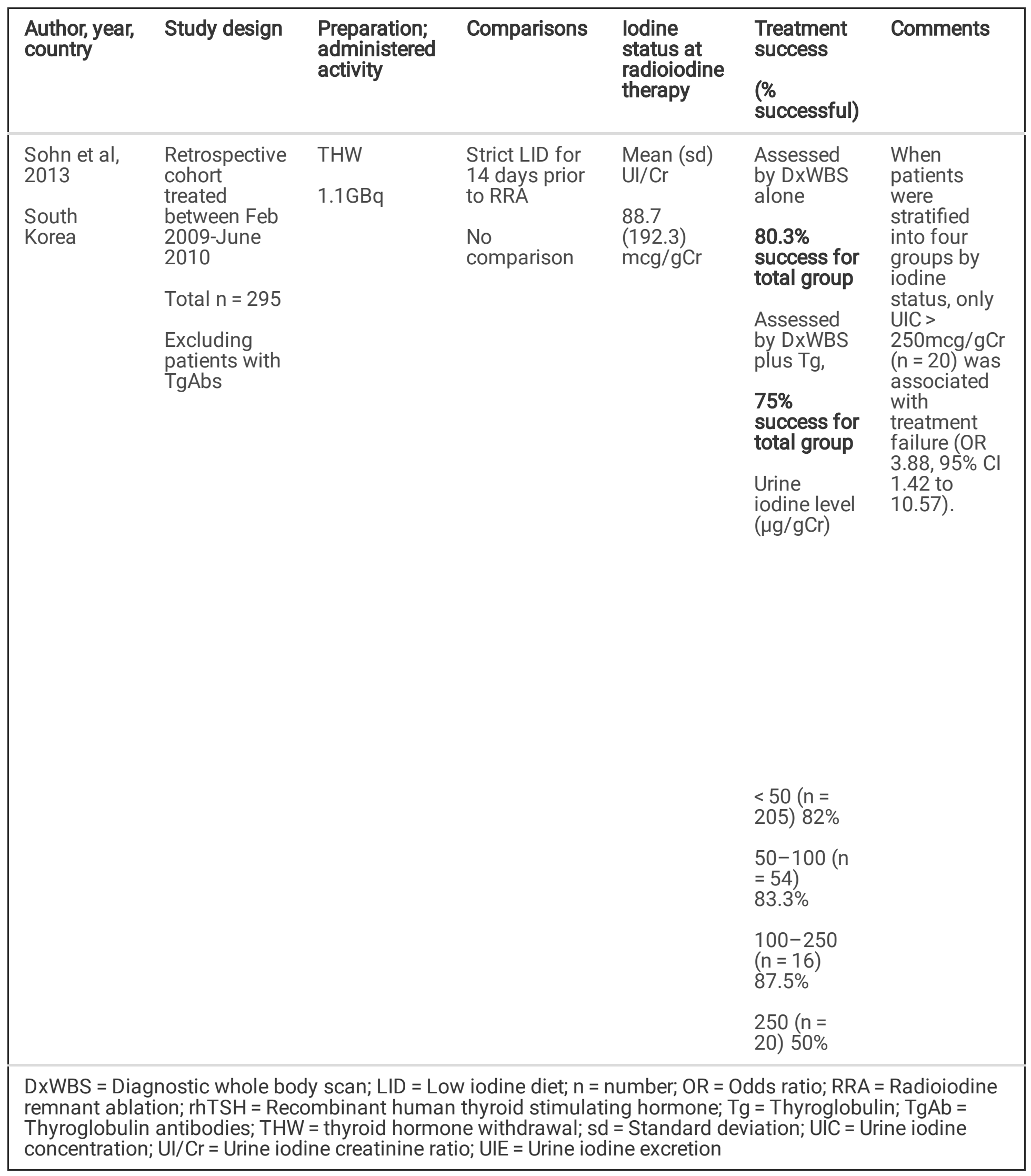

\section{lodine status}

Only two studies $(21,22)$ summarised in Table 3 measured iodine status in all participants. Sohn et al(22) reported a reduction in success rates only in people with very high iodine status, although Tala Jury et al(21) did 
not observe a similar reduction. Non-randomised $(7,8,23,24)$ and randomised $(25,26)$ studies have shown that advice to follow an LID for one to two weeks can lower UIC to $<100 \mathrm{mcg} / \mathrm{I}$. However, iodine status is not routinely measured prior to RRA in the UK(13) and we were unable to assess whether patients reduced their iodine status or whether iodine status itself was associated with treatment success. It is also unknown whether patients in C1 reduced high iodine foods despite being given no specific LID advice. There is readily available information online about the LID(27) which patients could have accessed. We have conducted qualitative work that suggests patients not given advice to follow a LID may still reduce iodine intake(28).

\section{Other strengths and limitations}

This is the first study conducted in the UK to investigate whether advice to follow a LID prior to RRA affects treatment success in DTC. Ideally, the only difference in treatment across centres would have been in the dietary advice given. However, unexpectedly, there was evidence for a difference between the centres in ${ }^{131}$ I administered activity with fewer patients in $\mathrm{C} 3$ having higher administered activity. The fully adjusted model corrected for activity but there were also differences in preparation and assessment methods between centres that we were not aware of when designing the study. Although we do not consider these to be factors that would substantially affect treatment success(29), this meant we were not comparing identical practices. Given that evidence from other studies seems to indicate that following a LID prior to RRA does not confer substantial benefits in terms of outcomes, the differences between centres may have masked any small benefits from a LID.

\section{Recommendations for research and practice}

This study forms part of a larger overall project examining advice to follow a LID prior to ablation. We have conducted a qualitative study investigating the impact of the advice on patients(28) and a survey of practice regarding the use of the LID in the UK(13). This retrospective study indicates that routinely collected data cannot be used in the UK to determine whether advice to follow an LID has an impact on treatment success due to centre-level differences on top of differences in LID advice. In the UK, it is unclear whether current LID advice successfully lowers iodine intake or iodine status and, globally, it remains unclear as to whether low iodine status improves treatment success. The flaws in our retrospective study indicate that there is a need for an RCT or, given that the LID is widely used in clinical practice, a large well-controlled prospective observational study to determine 1) whether LID advice lowers iodine status and 2) whether lowering iodine status has an impact on treatment success rates.

\section{Conclusion}

We conducted this retrospective review to determine if following a low iodine diet for 1 or 2 weeks prior to RRA for differentiated thyroid cancer contributed to treatment success. In common with other retrospective studies, we found no evidence that such advice impacted on success rate. However, differences in preparation and assessment meant we were not comparing identical practices across centres and the results should be treated as exploratory. There is no immediate need to change practice regarding the LID in the centres giving different dietary advice, but there is a need for greater harmonisation in overall treatment, drawing on evidence from recent trials(30). We believe that adequately powered well-controlled prospective studies and, potentially, an RCT, are required to confirm the role of a LID prior to RRA on the outcomes of treatment. 


\section{List Of Abbreviations}

ATA = American Thyroid Association

$95 \% \mathrm{Cl}=95 \%$ Confidence interval

DTC $=$ Differentiated thyroid cancer

DxWBS = Diagnostic whole-body scan

LID $=$ Low iodine diet

$\mathrm{OR}=$ Odd ratio

PaWBS = Post ablation whole body scan

RCT = Randomised controlled trial

rhTSH $=$ Recombinant human thyroid stimulating hormone

RRA = Radioiodine remnant ablation

$\mathrm{Tg}=$ Thyroglobulin

TgAb = Thyroglobulin antibodies

Tg-IA = Thyroglobulin immunometric assays

Tg-RIA = Thyroglobulin radioimmunoassays

THW = Thyroid hormone withdrawal

TSH $=$ Thyroid stimulating hormone

UIC = Urine iodine concentration

$\mathrm{UI} / \mathrm{Cr}=$ Urine iodine creatinine ratio

UIE = Urine iodine excretion

US = ultrasound

\section{Declarations}

\section{Acknowledgements}

Not applicable

Ethical approval and consent statement 
The UK National Health Service Health Research Authority (https://www.hra.nhs.uk/) which oversees the research ethics process within the UK, advised that, according to national regulations, this study was a Clinical Surveillance Audit and did not need a review by a Research Ethics Committee. The study was formally approved locally as a Service Evaluation by The Royal Liverpool and Broadgreen University Hospital Trust, Liverpool, England and as an Audit by University Hospital Bristol and Weston NHS Trust, Bristol, England and by Velindre University NHS Trust, Cardiff, Wales and, as such, information officers and audit mangers at each Trust determined that consent to participate was not needed on the basis that the study used anonymised data. Administrative permissions to extract anonymised clinical data used in the study was granted by each Trust to local staff with substantive or honorary contracts.

\section{Consent for publication}

Not applicable

\section{Availability of data}

The datasets used and/or analysed during the current study are available from the corresponding author on reasonable request.

\section{Competing interest statement}

IHS is the lead and LM is a member of the UK Low lodine Diet Working Group. The remaining authors have nothing to declare.

\section{Funding sources}

This study was funded by the NIHR Bristol Biomedical Research Centre at University Hospitals Bristol and Weston NHS Foundation Trust and the University of Bristol. The views expressed are those of the authors and not necessarily those of the NIHR or the Department of Health and Social Care.

\section{Author contributions}

$\mathrm{CE}, \mathrm{MB}, \mathrm{LM}, \mathrm{SV}$, IHS, GM, GH, AN and CA designed the study; CE and GH undertook literature research. Data extraction was performed by CE, MB, LM, IHS, SV and GM. Statistical analysis was conducted by KIH and LH under supervision of SL. CE drafted the initial manuscript which was revised and edited by all authors.

\section{References}

1. Deng Y, Li H, Wang M, Li N, Tian T, Wu Y, et al. Global Burden of Thyroid Cancer From 1990 to 2017. JAMA Network Open. 2020;3(6):e208759-e.

2. Cancer Research UK. Thyroid cancer incidence statistics: Cancer Research UK; 2020 [updated March 2020. Available from: https://www.cancerresearchuk.org/health-professional/cancer-statistics/statistics-bycancer-type/thyroid-cancer/incidence\#heading-Zero.

3. Perros P, Boelaert K, Colley S, Evans C, Evans RM, Gerrard Ba G, et al. Guidelines for the management of thyroid cancer. Clinical Endocrinology. 2014;81:1-122. 
4. Pacini F, Castagna MG. Diagnostic and therapeutic use of recombinant human TSH (rhTSH) in differentiated thyroid cancer. Best Practice \& Research Clinical Endocrinology \& Metabolism. 2008;22(6):1009-21.

5. Luster M, Clarke SE, Dietlein M, Lassmann M, Lind P, Oyen WJ, et al. Guidelines for radioiodine therapy of differentiated thyroid cancer. European journal of nuclear medicine and molecular imaging. 2008;35(10):1941-59.

6. Ju DL, Park YJ, Paik H-Y, Kim M-J, Park S, Jung KY, et al. Dietary evaluation of a low-iodine diet in Korean thyroid cancer patients preparing for radioactive iodine therapy in an iodine-rich region. Nutrition Research and Practice. 2016;10(2):167-74.

7. Lee M, Lee YK, Jeon TJ, Chang HS, Kim BW, Lee YS, et al. Low iodine diet for one week is sufficient for adequate preparation of high dose radioactive iodine ablation therapy of differentiated thyroid cancer patients in iodine-rich areas. Thyroid. 2014;24(8):1289-96.

8. Lim CY, Kim JY, Yoon MJ, Chang HS, Park CS, Chung WY. Effect of a Low lodine Diet vs. Restricted lodine Diet on Postsurgical Preparation for Radioiodine Ablation Therapy in Thyroid Carcinoma Patients. Yonsei Med J. 2015;56(4):1021-7.

9. Li JH, He ZH, Bansal V, Hennessey JV. Low iodine diet in differentiated thyroid cancer: a review. Clinical Endocrinology. 2016;84(1):3-12.

10. Sawka AM, Ibrahim-Zada I, Galacgac P, Tsang RW, Brierley JD, Ezzat S, et al. Dietary iodine restriction in preparation for radioactive iodine treatment or scanning in well-differentiated thyroid cancer: a systematic review. Thyroid. 2010;20(10):1129-38.

11. Public Health England. NDNS: results from years 7 and 8 (combined): UK Government; 2018 [Available from: https://www.gov.uk/government/statistics/ndns-results-from-years-7-and-8-combined.

12. UK Low lodine Diet Working Group. The Low lodine Diet 2018 [Available from: http://www.btfthyroid.org/images/documents/Low_lodine_Diet_Factsheet.pdf.

13. England CY, Moss L, Beasley M, Haupt-Schott I, Herbert G, Atkinson C. A Survey of UK Centres on Low lodine Diet Recommendations prior to Radioiodine Ablation Therapy for Differentiated Thyroid Cancer. European Thyroid Journal. 2019.

14. Haugen BR, Alexander EK, Bible KC, Doherty GM, Mandel SJ, Nikiforov YE, et al. 2015 American Thyroid Association Management Guidelines for Adult Patients with Thyroid Nodules and Differentiated Thyroid Cancer: The American Thyroid Association Guidelines Task Force on Thyroid Nodules and Differentiated Thyroid Cancer. Thyroid. 2016;26(1):1-133.

15. Spencer $\mathrm{C}$, Fatemi S. Thyroglobulin antibody (TgAb) methods - Strengths, pitfalls and clinical utility for monitoring TgAb-positive patients with differentiated thyroid cancer. Best Practice \& Research Clinical Endocrinology \& Metabolism. 2013;27(5):701-12.

16. Sobin LH, Gospodarowicz MK, Wittekind C, Cancer IUA. TNM Classification of Malignant Tumours, 7th Edition. Chichester, West Sussex, UK: Wiley-Blackwell; 2009.

17. Rotteveel-de Groot DM, Ross HA, Janssen MJR, Netea-Maier RT, Oosting JD, Sweep FCGJ, et al. Evaluation of the highly sensitive Roche thyroglobulin II assay and establishment of a reference limit for thyroglobulinnegative patient samples. Pract Lab Med. 2016;5:6-13. 
18. Mallick U, Harmer C, Hackshaw A, Moss L. lodine or Not (IoN) for Low-risk Differentiated Thyroid Cancer: The Next UK National Cancer Research Network Randomised Trial following HiLo. Clinical Oncology. 2012;24(3):159-61.

19. Maxon HR, Thomas SR, Boehringer A, Drilling J, Sperling MI, Sparks JC, et al. Low iodine diet in I-131 ablation of thyroid remnants. Clin Nucl Med. 1983;8(3):123-6.

20. Pluijmen MJ, Eustatia-Rutten C, Goslings BM, Stokkel MP, Arias AM, Diamant M, et al. Effects of low-iodide diet on postsurgical radioiodide ablation therapy in patients with differentiated thyroid carcinoma. Clin Endocrinol (Oxf). 2003;58(4):428-35.

21. Tala Jury HP, Castagna MG, Fioravanti C, Cipri C, Brianzoni E, Pacini F. Lack of association between urinary iodine excretion and successful thyroid ablation in thyroid cancer patients. J Clin Endocrinol Metab. 2010;95(1):230-7.

22. Sohn SY, Choi JY, Jang HW, Kim HJ, Jin SM, Kim SW, et al. Association between excessive urinary iodine excretion and failure of radioactive iodine thyroid ablation in patients with papillary thyroid cancer. Thyroid. 2013;23(6):741-7.

23. Park JT, Hennessey JV. Two-week low iodine diet is necessary for adequate outpatient preparation for radioiodine rhTSH scanning in patients taking levothyroxine. Thyroid: official journal of the American Thyroid Association. 2004;14(1):57-63.

24. Padovani RP, Maciel RMB, Kasamatsu TS, Freitas BCG, Marone MMS, Camacho CP, et al. Assessment of the Effect of Two Distinct Restricted lodine Diet Durations on Urinary lodine Levels (Collected over $24 \mathrm{~h}$ or as a Single-Spot Urinary Sample) and $\mathrm{Na}(+) / \mathrm{I}(-)$ Symporter Expression. European Thyroid Journal. 2015;4(2):99105.

25. Morsch EP, Vanacor R, Furlanetto TW, Schmid H. Two weeks of a low-iodine diet are equivalent to 3 weeks for lowering urinary iodine and increasing thyroid radioactive iodine uptake. Thyroid. 2011;21(1):61-7.

26. Wan Sohaimi WF, Abdul Manap M, Kasilingam L, Ab Hamid S-A, Mat Nawi N, Abd Hamid MHN. Randomised controlled trial of one week strict low-iodine diet versus one week non-specified low iodine diet in differentiated thyroid carcinoma: Research Center for Nuclear Medicine (Tehran University of Medical Sciences); 2019. 99-105 p.

27. Unknown. Thyroid Cancer Group. Thyroid Cancer. UK: MacMillan Cancer Support; 2019.

28. Herbert G, Searle A, England CY, Ness A, Beasley M, Haupt-Schott I, et al. Experiences of low iodine diets in the treatment of differentiated thyroid cancer with radioactive iodine ablation therapy. Clinical Nutrition ESPEN. 2020.

29. Mallick U, Harmer C, Yap B, Wadsley J, Clarke S, Moss L, et al. Ablation with Low-Dose Radioiodine and Thyrotropin Alfa in Thyroid Cancer. 2012;366(18):1674-85.

30. Wadsley J, Dehbi H-M, Mallick U, Newbold K, Harmer C, Hackshaw A. Recurrence rates after low dose radioiodine ablation for differentiated thyroid cancer within the NCRI HiLo trial. NCRI Cancer Conference abstracts. 2018.

\section{Supplementary Files}

This is a list of supplementary files associated with this preprint. Click to download. 
- Supplementarytables.docx

Page 20/20 